\title{
BMJ Open Association between health behaviours and cardiometabolic dysregulation: a population-based survey among healthy adults in Hong Kong
}

\author{
Esther Yee Tak Yu (D) , ${ }^{1}$ Caitlin Hon Ning Yeung, ${ }^{1}$ Eric Yuk Fai Wan (D) , , \\ Eric Ho Man Tang, ${ }^{1}$ Carlos King Ho Wong (D) , ${ }^{1,2}$ Bernard Man Yung Cheung, ${ }^{3}$ \\ Cindy Lo Kuen Lam ${ }^{1}$
}

To cite: Yu EYT, Yeung CHN, Wan EYF, et al. Association between health behaviours and cardiometabolic dysregulation: a population-based survey among healthy adults in Hong Kong. BMJ Open 2021;11:e043503. doi:10.1136/ bmjopen-2020-043503

- Prepublication history and supplemental material for this paper is available online. To view these files, please visit the journal online (http://dx.doi. org/10.1136/bmjopen-2020043503).

Received 14 August 2020 Accepted 02 June 2021

Check for updates

(c) Author(s) (or their employer(s)) 2021. Re-use permitted under CC BY-NC. No commercial re-use. See rights and permissions. Published by BMJ.

${ }^{1}$ Department of Family Medicine and Primary Care, The University of Hong Kong, Hong Kong ${ }^{2}$ Department of Pharmacology and Pharmacy, The University of Hong Kong, Hong Kong ${ }^{3}$ Department of Medicine, The University of Hong Kong, Hong Kong

Correspondence to Dr Esther Yee Tak Yu; ytyu@hku.hk

\section{ABSTRACT}

Objective To explore the association between cardiometabolic dysregulation, an integral component of allostatic load, and health risk behaviours (HRBs) of the Hong Kong healthy adult population.

Design Secondary analysis of cross-sectional anonymous data.

Setting Data on sociodemographics, self-reported health status, HRBs and biomarkers were extracted from the Hong Kong Population Health Survey 2014/2015.

Participants One thousand five hundred and fiftyone participants aged 18-64 years without selfreported diagnoses of hypertension, diabetes mellitus, hyperlipidaemia, cardiovascular disease, cognitive impairment or cancer.

Primary outcome measures Cardiometabolic dysregulation index (CMDI), ranging from 0 to 6 , was calculated by counting the number of biomarkers including systolic blood pressure, diastolic blood pressure, waist to hip ratio, glycated haemoglobin, total cholesterol to high-density lipoprotein cholesterol ratio, and triglycerides that were above the respective normal level suggested by international guidelines and literature. HRBs including smoking, dietary habits and sleeping hours were collected by self-report questionnaire. Alcohol consumption was assessed by the 10-item Alcohol Use Disorders Identification Test, while physical activity level was measured using the Global Physical Activity Questionnaire. A composite HRB score, ranging from 0 to 5 , was calculated as the cumulative number of HRBs. The effect of HRB on CMDI was evaluated by negative binomial regression with adjustment for socioeconomic status, health awareness and comorbidities of the participants.

Results The mean CMDI of the studied population was 1.6; $29.5 \%$ had a CMDI of 0 , whereas $1.5 \%$ had a CMDI of 6 . Significant difference was observed in mean CMDI between gender and different age groups. Sleeping less than 6 hours (incidence rate ratio (IRR) $=1.26, p<0.001$ ), smoking (IRR=1.15, $p=0.027$ ), insufficient physical activity (IRR=1.12, $\mathrm{p}=0.007)$ and higher composite HRB score (IRR=1.12, 95\% $\mathrm{Cl} 1.06$ to 1.18) were significantly associated with higher CMDI.

Conclusion Smoking, physical inactivity and inadequate sleep —an essential yet often overlooked health
Strengths and limitations of this study

- This is the first study to explore the association between cardiometabolic dysregulation, an integral component of allostatic load, and various health risk behaviours, as well as their combined effect, among the Hong Kong healthy adult population, using data from a territory-wide health survey of a large representative sample.

- We measured cardiometabolic dysregulation using a practical yet robust index, evaluated health risk behaviours with standardised measurement and considered a comprehensive range of socioeconomic factors as confounders.

- As a cross-sectional study, the relationship between cardiometabolic dysregulation and development of clinically important health outcomes such as diabetes mellitus, cardiovascular events and mortality cannot be examined.

- The operationalisation of the cardiometabolic dysregulation index in our study did not include biomarkers from the neuroendocrine, inflammatory or immunology systems and hence could not reflect the overall allostatic load of the study participants.

- Health risk behaviours and socioeconomic information were collected with self-administered questionnaire, and recall or social desirability bias might occur.

behaviour-were associated with higher CMDI in the Hong Kong healthy adult population.

\section{INTRODUCTION}

Cumulative stress from persistent socioeconomic disadvantage, psychosocial adversities and health risk behaviours (HRBs) across life course has been linked to physiological dysregulation, chronic diseases and increased mortality. ${ }^{1}$ Allostatic load (AL) provides a useful framework for conceptualising the overall physiological 'wear-and-tear' on one's body due to chronic life stress. ${ }^{2}$ Allostasis 
refers to the dynamic, adaptive regulatory process that maintains homeostasis during exposure to stressors. ${ }^{3}$ Repeated cycles of activation and deactivation of allostasis over time lead to neuroendocrine system maladaptation, which results in secondary physiological dysregulation in the metabolic, cardiovascular and inflammatory systems. AL captures the cumulative, multisystem effects of stress before onset of diseases (ie, allostatic overload) using an array of biological markers at increased-risk ranges. ${ }^{4}$ Previous research demonstrated that elevated AL predicted adverse health outcomes in later life, ${ }^{5}$ including physical and cognitive decline ${ }^{6}$ malignant neoplasms, cardiometabolic and respiratory diseases, ${ }^{7}$ depressive symptoms, ${ }^{8}$ and all-cause mortality, ${ }^{9}$ better than any individual biomarker or the metabolic syndrome. ${ }^{56}$ Hence, assessment of AL allows early identification of people at increased health risks when the physiological dysregulations are still reversible, such that timely interventions, such as exercise, ${ }^{10}$ drug therapy, diet and psychological intervention, ${ }^{11}$ can be provided to prevent adverse health outcomes.

To mitigate the health consequences of elevated AL, identification of its modifiable antecedents is imperative. In a recent systematic review, Suvarna $e t a l^{12}$ found that different HRBs contributed to AL variably. While there is strong evidence on the relationship between physical inactivity and AL, evidence on the relationships of other HRBs and AL is less consistent. ${ }^{12}{ }^{13}$ While a number of studies supported the link between smoking and high AL, ${ }^{14-16}$ Petrovic et al found only a weak and insignificant association, whereas Gallo et $a l^{1317}$ found no relationship between them. Alcohol consumption had been identified as a predictor of both high and low AL. ${ }^{13}{ }^{17-19}$ Although van Draanen $e t a t^{20}$ showed that high AL was associated with consumption of fast foods, sugar-sweetened beverages and artificially sweetened beverages, Mattei $e t a l^{21}$ found that the association between increased sweet consumption and heightened AL was only significant among participants with diabetes. Poor sleep quality, sleep pattern variability, inadequate sleep as well as excessive sleep had all been related to increased AL. ${ }^{22-25}$ It is possible that the results from the different studies could not be directly compared due to the different ways which HRBs and AL were measured.

The major challenge in measuring AL to screen and monitor an individual's health risk and guide preventive care in both routine clinical practice and research lies in its operationalisation. AL is typically operationalised as a single composite index of biological markers in the increased-risk ranges, referred to as the allostatic load index (ALI). Controversies remain in which biomarkers to include, how to measure, combine and weigh them, and with which statistical analytic techniques. ${ }^{1}$ The original ALI comprised 10 biomarkers from the neuroendocrine, cardiovascular and metabolic systems. ${ }^{7}$ However, this preliminary operational definition of ALI has not been adopted as the gold standard.$^{26} \mathrm{~A}$ wide range of biomarkers representing five main biological systems have been used in the literature, with the number of biomarkers used to calculate ALI ranging from 4 to $24{ }^{27-29} \mathrm{~A}$ total of 39 biomarkers were used to calculate AL indices in the 26 studies included in Suvarna et $a l \mathrm{~s}^{12}$ review, where only $9(23.1 \%)$ were common, namely systolic blood pressure (SBP), diastolic blood pressure (DBP), glycated haemoglobin (HbA1c), high-density lipoprotein cholesterol (HDL-C), total cholesterol (TC), body mass index (BMI), waist to hip ratio (WHR), triglycerides (TG) and C-reactive protein/high sensitivity C-reactive protein. The other biomarkers hardly overlapped. Furthermore, studies have pointed out that neuroendocrine biomarkers, despite their centrality in the conceptualisation of $\mathrm{AL}$, are inconsistent and impractical to measure in clinical settings due to their circadian rhythmicity. ${ }^{20}$ Differentiation between circadian changes in neuroendocrine parameters and transient changes versus long-term response to stress cannot be readily made from spot sampling. Moreover, considerable heterogeneity and an unusually broad range were observed in the cut-off points of each biomarker for defining high AL used in previous studies. Many studies used quartile-based cut-offs as the 'high-risk' thresholds, which were specific for the studied population and precluded their use in other populations. ${ }^{2}$

Conversely, most cardiovascular and metabolic biomarkers, which are essential constituents of $\mathrm{AL}$ measuring the secondary physiological dysregulation resulting from chronic stress, are routinely available in daily clinical practice and commonly measured in population-based studies. The presence of individual or combination of cardiometabolic risk factors has been termed 'cardiometabolic dysregulation' (CMD) or 'cardiometabolic risk' in the literature; a composite CMD score has been conveniently calculated as the simple count of cardiometabolic biomarkers in the respective high-risk ranges according to clinical criteria, which has the potential to be applied to different populations and would allow comparison across different studies. ${ }^{31-33}$ Similar to AL, increased CMD was associated with stressful and traumatic life experiences,${ }^{34}$ cognitive decline, ${ }^{32}$ and HRBs. ${ }^{31}{ }^{33}$ Shi et $a l^{31}$ demonstrated that more healthy lifestyle behaviours, including abstinence from smoking, regular exercise, sufficient fruit and vegetable intake, less meat intake, modest alcohol intake and less television viewing time, were associated with reduced CMD among two generations of adults in the USA and Canada. However, no study has yet examined the relationship between CMD and various HRBs among the younger healthy Asian adult population.

This study aims to explore the association between the predisease CMD and HRBs of the Hong Kong healthy adult population, using readily available biomarkers in routine clinical setting and cut-off levels above the respective 'normal' levels recommended by international guidelines and literature. 


\section{METHODS}

\section{Study design and subject sampling}

Secondary analysis was conducted on data extracted from the Population Health Survey 2014/2015, a territory-wide cross-sectional survey conducted by the Department of Health of the Hong Kong Special Administrative Region Government. Detailed sampling method of the Population Health Survey 2014/2015 has been reported by the Department of Health. ${ }^{35}$ In brief, the Population Health Survey 2014/2015 adopted systematic replicated sampling to duplicate the land-based, non-institutional population excluding domestic helpers and visitors of Hong Kong. The survey successfully recruited 12022 participants aged 15 years or above from 5435 domestic households as the representative sample of the Hong Kong general population. Each participant provided written consent and completed a questionnaire collecting information on sociodemographics, self-reported health status and HRBs. Among them, 2347 participants aged 15-84 years were further randomly sampled, stratified by age and gender, to undergo health examination including blood pressure, waist and hip circumference measurement, and blood tests for HbAlc and full lipid profile. In this study focusing on younger healthy adults, only participants aged between 18 and 64 years who completed the questionnaire and underwent the health examination were included; 416 participants aged below 18 years or above 64 years were excluded. To evaluate the cardiometabolic dysregulation index (CMDI) as a predisease health risk indicator, 380 participants with self-reported doctor diagnosis or taking medication(s) which would affect the spot value of the biomarkers, including diabetes mellitus, hypertension or high blood cholesterol, or having cardiovascular disease (coronary heart disease and stroke), renal disease, thyroid disease, cancer or cognitive impairment, were further excluded. Online supplemental figure 1 shows the inclusion process of the 1551 participants in this study.

\section{Cardiometabolic dysregulation index}

CMDI was operationalised as a simple count of the number of biomarkers that were above the respective normal level recommended by international clinical guidelines and literature (online supplemental table 1). The six selected biomarkers and their respective cut-offs were $\mathrm{SBP} \geq 130 \mathrm{~mm} \mathrm{Hg}, \mathrm{DBP} \geq 80 \mathrm{~mm} \mathrm{Hg}$, WHR $\geq 0.9$ for men or $\geq 0.8$ for women, HbAlc $\geq 5.7 \%$, TC to HDL-C ratio $\geq 4.5$ for men or $\geq 4.0$ for women, and TG $\geq 1.7 \mathrm{mmol} / \mathrm{L}$. As a numeric scale ranging from 0 to 6 , a higher CMDI score reflects higher level of CMD.

\section{Health behaviours}

Five health behaviours were evaluated, namely smoking, alcohol consumption, physical activity (PA), dietary habit and sleep. Smoking status was classified into "current smoker', 'former chronic smoker' or 'never smoked'. Alcohol consumption habit was assessed by the 10-item Alcohol Use Disorders Identification Test (AUDIT). ${ }^{36}$
Drinking status was categorised into 'non-drinker', 'lowrisk drinking' (ie, AUDIT score $<8$ ) or 'harmful drinking' (ie, AUDIT score $\geq 8$ ) ${ }^{3637}$ PA level was measured using the Global Physical Activity Questionnaire (GPAQ), which collects PA level at work, during recreational activities and travelling. ${ }^{38}$ PA level was considered 'sufficient' if the participant performed at least $150 \mathrm{~min}$ of moderateintensity PA, $75 \mathrm{~min}$ of vigorous-intensity PA or at least 600 metabolic equivalent-minutes per week. ${ }^{39}$ Two questions were used to assess dietary habits, where one question asked for the frequency of vegetables and/or fruits per day and the other question counted the amount of vegetables and/or fruits per intake. Fruit and vegetable consumption per day was categorised into ' $<3$ servings', '3-4 servings' or ' $\geq 5$ servings'. Daily intake of $\geq 5$ servings $(400 \mathrm{~g})$ of vegetables and/or fruits was considered as 'adequate'. ${ }^{40}$ Sleep was evaluated in terms of self-rated sleep quality ('poor', 'fair' or 'well') and sleeping hours, which were categorised into ' $<6$ hours', ' $6-8$ hours' or ' $\geq 9$ hours'. ${ }^{41}{ }^{42}$ A composite HRB score ranging from 0 to 5 was computed as the sum of HRBs of each participant, including current smoker, harmful drinking (AUDIT score $\geq 8)$, insufficient $\mathrm{PA}$, inadequate fruit/vegetable consumption $(<5$ servings per day) and inadequate sleep $(<6$ hours per day).

\section{Covariates}

Other potential stressors including socioeconomic status, health awareness and comorbidities of the participants were considered as confounders. Socioeconomic characteristics included age, gender, marital status, monthly household income, educational level and working status. Marital status was classified into 'single' or 'married', where 'single' included participants who were never married, divorced, separated and widowed. Monthly household income was self-reported and classified into ' $<50 \%$ Hong Kong median', 'between 50\% and the Hong Kong median' or 'above Hong Kong median', based on the Hong Kong median monthly household income in the second quartile of year 2015 (HK\$24 500). Highest educational level attained was categorised into 'secondary education or below' or 'tertiary education or above'. Working status was coded as 'active' if the subject had a full-time or part-time job in the past 7 days, otherwise as 'inactive'. Health awareness was implied from the practice of regular health check. Physical comorbidities which might indirectly influence AL or CMD included asthma, chronic obstructive pulmonary disorder, respiratory diseases, liver disease, stomach disease, anaemia, musculoskeletal diseases, immunological disorders, skin disorders or Parkinson disease, while mental comorbidities included depression, anxiety disorder or schizophrenia.

\section{Statistical analysis}

Multiple imputation by chained equation was deployed to handle missing covariates. Each missing value was imputed five times by multivariate chained equations with the use of all other known covariates. ${ }^{43}$ Each imputed data set was 
used to perform analysis individually and the results were pooled together by Rubin's combination rule. ${ }^{44}$

Descriptive statistics were used to present the characteristics of the participants, including mean and SD for continuous variables and proportion and number of participants for categorical variables. The CMDI by different age groups and gender was compared by oneway analysis of variance with post-hoc Tukey honestly significant difference test, given that age and gender were well-recognised confounders of increased risks of CMDI biomarkers in the literature. ${ }^{45-48}$ The incidence rate ratios (IRRs) for the individual and combined effects of HRBs on CMDI were evaluated by negative binomial regression adjusted by gender, age, marital status, educational level, household income, working status, physical comorbidities, mental comorbidities and regular health check. Multicollinearity was examined by variance inflation factor. Sensitivity analysis was performed using completecase analysis, where participants without complete data on covariates were excluded from the analysis.

All statistical analyses were performed by Stata V.16.0. All tests of significance were two-tailed and $\mathrm{p}$ value $<0.05$ was considered statistically significant.

\section{Patient and public involvement}

Participants were not involved in setting the research question or the outcomes measures, designing the investigation, or interpreting the data. There was no plan to involve participants in the dissemination of the results.

\section{RESULTS}

Table 1 presents the characteristics of participants; data completion rates are listed in online supplemental table 2. Of the participants, $47.2 \%$ were male and the mean age was $37.5(\mathrm{SD}=12.9)$. Of the participants, $195(12.6 \%)$ were current smokers, among whom $30(15.4 \%)$ were social smokers (ie, did not smoke daily). The average number of cigarettes smoked by the 165 current daily smokers was 12.26 ( $\mathrm{SD}=7.06$, range $1-40$ ). Of the participants, $4.4 \%$ exhibited harmful alcohol use. While $39.2 \%$ of the participants achieved sufficient PA level, only $6.3 \%$ consumed the recommended five servings of fruits and/or vegetables daily. Of the participants, $9.8 \%$ reported having less than 6 hours of sleep per day.

The mean CMDI was $1.6(\mathrm{SD}=1.5)$. Table 2 presents the distribution of CMDI by age and gender. Of the participants, $29.5 \%$ had a CMDI of 0, including 34 participants $(6.9 \%)$ aged between 45 and 64 . Twenty-three participants $(1.5 \%)$ had a CMDI of 6 , among whom one subject was younger than 30 years. The mean CMDI increased significantly from $0.8(\mathrm{SD}=1.0)$ in participants between 18 and 29 years to $1.5(\mathrm{SD}=1.4)$ and $2.6(\mathrm{SD}=1.5)$ among those aged 30-44 and 45-64 years, respectively. Significant differences were observed between the mean CMDI of men and women. The mean CMDI by different age groups and gender is compared in figure 1.
Table 1 Characteristics of participants $(\mathrm{N}=1551)$

\begin{tabular}{ll}
\hline Factors & $\begin{array}{l}\text { Participants, } \\
\text { mean } \pm \text { SD/n (\%) }\end{array}$ \\
\hline $\begin{array}{l}\text { Socioeconomic factors } \\
\text { Male }\end{array}$ & $732(47.2)$ \\
Age & $37.5 \pm 12.9$ \\
$\quad$ Marital status (married) & $826(53.3)$ \\
$\quad \begin{array}{l}\text { Tertiary education or above } \\
\text { Household income }\end{array}$ & $703(45.3)$ \\
$\begin{array}{l}\text { Above the Hong Kong median } \\
\text { household income }\end{array}$ & $1060(68.3)$ \\
\hline
\end{tabular}

Between 50\% and the Hong Kong 358 (23.1) median household income

\begin{tabular}{ll}
$\begin{array}{ll}\text { Below } 50 \% \text { Hong Kong median } \\
\text { household income }\end{array}$ & $133(8.6)$ \\
\hline Active working status $\dagger$ & $1172(75.6)$ \\
\hline $\begin{array}{l}\text { Health conditions } \\
\text { Physical comorbidities }\end{array}$ & $191(12.3)$ \\
\hline Mental comorbidities & $19(1.2)$ \\
\hline Regular health check practice & $470(30.3)$ \\
\hline Lifestyle factors & \\
\hline Smoking status & \\
\hline Never smoked & $1230(79.3)$ \\
\hline Former chronic smoker & $126(8.1)$ \\
\hline $\begin{array}{l}\text { Current smoker } \\
\text { Alcohol consumption pattern }\end{array}$ & $195(12.6)$ \\
\hline Non-drinker & $418(27.0)$ \\
\hline Low-risk drinking (AUDIT score <8) & $1064(68.6)$ \\
Harmful drinking (AUDIT score $\geq 8)$ & $69(4.4)$
\end{tabular}

Physical activity

Sufficient physical activity level by 608 (39.2) WHO recommendation

Dietary habit

Fruit/vegetable consumption per day

$<3$ servings

$1151(74.2)$

$3-4$ servings $302(19.5)$

$\geq 5$ servings

$98(6.3)$

Sleep

Good sleep quality $\quad 886(57.1)$

Sleep hours $6.9 \pm 1.1$

$<6$ hours $152(9.8)$

6-9 hours 1331 (85.8)

$\geq 9$ hours

$68(4.4)$

Composite health risk behaviour score (range 0-5)

$1.86 \pm 0.76$

Cardiometabolic dysregulation

$\begin{array}{ll}\mathrm{CMDI} \text { (range 0-6) } & 1.6 \pm 1.5 \\ \mathrm{SBP} \geq 130 \mathrm{~mm} \mathrm{Hg} & 194(12.5)\end{array}$

Continued 


\section{Table 1 Continued}

\begin{tabular}{ll} 
Factors & $\begin{array}{l}\text { Participants, } \\
\text { mean } \pm \text { SD/n (\%) }\end{array}$ \\
\hline $\mathrm{DBP} \geq 80 \mathrm{~mm} \mathrm{Hg}$ & $515(33.2)$ \\
WHR $\geq 0.8$ for women or $\geq 0.9$ for men & $664(42.8)$ \\
TC to HDL-C ratio $\geq 4.5$ for women or & $425(27.4)$ \\
$\geq 4.0$ for men & \\
TG $\geq 1.7 \mathrm{mmol} / \mathrm{L}$ & $226(14.6)$ \\
$\mathrm{HbA} 1 \mathrm{c} \geq 5.7 \%$ & $462(29.8)$ \\
\hline
\end{tabular}

Physical comorbidities included asthma, chronic obstructive pulmonary disorder, respiratory diseases, liver disease, stomach disease, anaemia, musculoskeletal diseases, immunological disorders, skin disorders or Parkinson disease. Mental comorbidities included depression, anxiety disorder or schizophrenia.

*The Hong Kong median household income in the second quartile of 2015 was HK\$24500.

†'Active working' is defined as having a full-time/part-time job in the past 7 days.

AUDIT, AlcoholUse Disorders Identification Test; CMDI, cardiometabolic dysregulation index; DBP, diastolic blood pressure; $\mathrm{HbA1c}$, glycated haemoglobin; HDL-C, high-density lipoprotein cholesterol; SBP, systolic blood pressure; TC, total cholesterol; TG, triglyceride; WHR, waist to hip ratio.

Table 3 presents the adjusted effect of HRBs on CMDI. Smokers (IRR=1.15, $\mathrm{p}=0.027$ ), participants with insufficient PA (IRR=1.12, $\mathrm{p}=0.007$ ) and participants with less than 6 hours of sleep (IRR $=1.26, p<0.001$ ) had significantly higher CMDI. On the other hand, consumption of vegetables, fruits or alcohol was not associated with CMDI. The interaction effect of gender and HRBs on CMD was insignificant. For sensitivity analysis, completecase analysis with 1522 participants was performed and found similar results. Higher composite HRB score was associated with higher CMDI (IRR=1.12, 95\% CI 1.06 to 1.18).

\section{DISCUSSION}

Our study demonstrated that the healthy adult population of Hong Kong between 18 and 64 years of age without preexisting cancer and cardiovascular or endocrine diseases had on average one or more cardiometabolic biomarkers above normal level (ie, mean CMDI=1.6). Around half of people aged between 45 and 64 years and $8.3 \%$ people aged between 18 and 29 years had a CMDI of 3 or above, suggestive of significant physiological dysregulation in cardiovascular and metabolic systems and increased health risk. Furthermore, our findings added evidence on the early harms of HRBs, that inadequate sleep, tobacco smoking, insufficient PA and higher number of HRBs were significantly associated with higher CMD.

The clinical value of AL assessment has been increasingly recognised in recent years, but its clinical application has been limited by inconsistencies in its measurement methods to date. In this study, we attempted to assess
AL by capturing secondary cardiovascular and metabolic dysregulations (ie, CMD) resulting from stress and consequent neuroendocrine system maladaptation using six routinely available biomarkers. Five out of the six included biomarkers were selected from the original ten AL biomarkers proposed by Seeman et a $a \tilde{l}$ and represented the most common and most robust biomarkers used in empirical literature. ${ }^{1}$ We used TC to HDL-C ratio to reflect lipoprotein metabolism dysregulation because the ratio had been shown to associate with more substantial alterations in metabolic indices predictive of cardiovascular disease and insulin resistance than considering their levels separately. ${ }^{49}$ TG level, a component of the metabolic syndrome and risk factor for cardiovascular disease, fatty liver and pancreatitis, was added to capture dysregulations in fat metabolism. ${ }^{50}$ For each biomarker, we defined increased risk as values above the respective normal cut-offs recommended by latest international clinical guidelines and/or literature to capture early changes before onset of diseases, which also corresponded to the mid-range of cut-off values used in previous studies on AL. ${ }^{4}$ Since there are no data on the relative importance of the biomarkers, we calculated the CMDI by an unweighted summation of the total number of biomarkers that were above the cut-offs. The validity and sensitivity of our method of CMDI calculation are well supported by the expected associations between CMDI, increasing age and various HRBs. ${ }^{31}$ Although the biomarkers used to assess CMDI overlap with the diagnostic criteria for metabolic syndrome, which dichotomises an individual to disease state (yes or no) based on the number of clinical criteria met, CMDI as a continuous variable does not label individuals with a diagnosis. Rather, an easy-to-assess CMDI informs clinicians and the individuals on the health risk of a person and need for more active preventive interventions, as well as allows monitoring of changes over time.

We found that around $10 \%$ of the Hong Kong healthy adult population reported inadequate sleep ( $<6$ hours), which was significantly associated with increased CMDI. The effects of sleep quantity and quality on AL, CMD and health were rarely explored. McEwen and Karatsoreos $^{51}$ proposed sleep disruption as key drivers of AL since good sleep is essential to let the body rest and repair damages. Prolonged inadequate sleep contributes to gradual changes in the regulatory set points that govern blood pressure, cholesterol, blood sugar and inflammatory activity, leading to disease pathophysiology. ${ }^{12}$ Associations between short sleep duration and various health problems had been established, including increased cardiometabolic risk score, odds of having metabolic syndrome, ${ }^{52}$ risk of upper respiratory tract infections ${ }^{53}$ and depression. ${ }^{54}$ McEwen and Karatsoreos's ${ }^{51}$ hypothesis was further supported by findings from Chen et al's ${ }^{24}$ study that sleep duration $<6$ hours, sleep apnoea, insomnia and diagnosed sleep disorder were all associated with high AL among adults in the USA. Clark $e t a l^{25}$ also found that the combination of short ( $<7$ hours) and disturbed sleep was associated 
Table 2 Sex and age distribution of cardiometabolic dysregulation index

\begin{tabular}{|c|c|c|c|c|}
\hline \multirow[b]{2}{*}{ Cardiometabolic dysregulation index } & \multicolumn{4}{|l|}{ Age group } \\
\hline & All ages & $18-29$ & $30-44$ & $45-64$ \\
\hline & \multicolumn{4}{|l|}{ Both sexes } \\
\hline & $(\mathrm{N}=1551)$ & $(n=530)$ & $(n=526)$ & $(n=495)$ \\
\hline Mean $\pm S D$ & $1.6 \pm 1.5$ & $0.8 \pm 1.0$ & $1.5 \pm 1.4$ & $2.6 \pm 1.5$ \\
\hline Median $\pm I Q R$ & $1 \pm 3$ & $0 \pm 1$ & $1 \pm 2$ & $2 \pm 3$ \\
\hline \multicolumn{5}{|l|}{ Proportion, \% (n) } \\
\hline 0 & $29.5(458)$ & $51.1(271)$ & $29.2(153)$ & $6.9(34)$ \\
\hline 1 & 26.4 (409) & $30.2(160)$ & $28.0(147)$ & $20.6(102)$ \\
\hline 2 & $18.0(280)$ & $10.5(55)$ & $19.9(105)$ & $24.2(120)$ \\
\hline 3 & $13.4(208)$ & $6.0(32)$ & $13.7(72)$ & $21.0(104)$ \\
\hline 4 & $7.0(109)$ & $1.5(8)$ & $5.8(31)$ & $14.3(71)$ \\
\hline 5 & $4.1(64)$ & $0.6(3)$ & $2.9(15)$ & $9.3(46)$ \\
\hline \multirow[t]{3}{*}{6} & $1.5(23)$ & $0.2(1)$ & $0.6(3)$ & $3.8(19)$ \\
\hline & \multicolumn{4}{|l|}{ Male } \\
\hline & $(n=732)$ & $(n=246)$ & $(n=259)$ & $(n=227)$ \\
\hline Mean \pm SD & $1.8 \pm 1.6$ & $0.9 \pm 1.2$ & $1.8 \pm 1.5$ & $2.7 \pm 1.6$ \\
\hline Median \pm IQR & $1 \pm 3$ & $1 \pm 1$ & $2 \pm 2$ & $3 \pm 2$ \\
\hline \multicolumn{5}{|l|}{ Proportion, \% (n) } \\
\hline 0 & $27.3(200)$ & 48.3 (119) & 24.6 (64) & $7.5(17)$ \\
\hline 1 & 23.5 (172) & $29.6(73)$ & $23.9(62)$ & $16.3(37)$ \\
\hline 2 & 17.6 (129) & $10.7(26)$ & $20.3(53)$ & $21.9(50)$ \\
\hline 3 & 17.2 (126) & $8.1(20)$ & $19.7(51)$ & $24.2(55)$ \\
\hline 4 & $7.2(53)$ & $2.0(5)$ & $6.4(17)$ & $13.8(31)$ \\
\hline 5 & $5.6(41)$ & $0.8(2)$ & $4.2(11)$ & $12.3(28)$ \\
\hline \multirow[t]{3}{*}{6} & $1.6(12)$ & $0.4(1)$ & $0.8(2)$ & $4.0(9)$ \\
\hline & \multicolumn{4}{|l|}{ Female } \\
\hline & $(\mathrm{n}=819)$ & $(\mathrm{n}=284)$ & $(\mathrm{n}=267)$ & $(\mathrm{n}=268)$ \\
\hline Mean \pm SD & $1.5 \pm 1.4$ & $0.7 \pm 0.9$ & $1.3 \pm 1.3$ & $2.5 \pm 1.5$ \\
\hline Median $\pm I Q R$ & $1 \pm 2$ & $0 \pm 1$ & $1 \pm 2$ & $2 \pm 2$ \\
\hline \multicolumn{5}{|l|}{ Proportion, \% (n) } \\
\hline 0 & 31.6 (259) & 53.5 (152) & $33.6(90)$ & $6.4(17)$ \\
\hline 1 & $29.0(237)$ & $30.7(87)$ & $31.9(85)$ & $24.2(65)$ \\
\hline 2 & $18.5(151)$ & $10.2(29)$ & $19.6(52)$ & $26.1(70)$ \\
\hline 3 & $10.0(82)$ & $4.2(12)$ & $7.9(21)$ & $18.3(49)$ \\
\hline 4 & $6.9(56)$ & $1.1(3)$ & $5.2(14)$ & $14.6(39)$ \\
\hline 5 & $2.8(23)$ & $0.4(1)$ & $1.5(4)$ & $6.6(18)$ \\
\hline 6 & $1.3(11)$ & $0(0)$ & $0.4(1)$ & 3.7 (10) \\
\hline
\end{tabular}

Significant difference in mean of cardiometabolic dysregulation index between different age groups and sex by one-way analysis of variance $(\mathrm{p}<0.05)$.

with higher AL in the older Danish population, and disturbed sleep might be an index of obesity-related disorder such as sleep apnoea. Conversely, our study did not show a significant relationship between CMDI and sleep quality or long sleep (9 hours or more) as demonstrated by Clark $e t a l{ }^{25}$ study. It was possible that the simple subjective rating of sleep quality used in our study was not sensitive enough to identify sleep impairment. Also, the presence of obstructive sleep apnoea, which is common among obese individuals who also have other concomitant cardiovascular and metabolic risk factors, has not been assessed in our study as a possible contributor to poor sleep quality. Given the prevalence and significance of sleep disorder in health, 


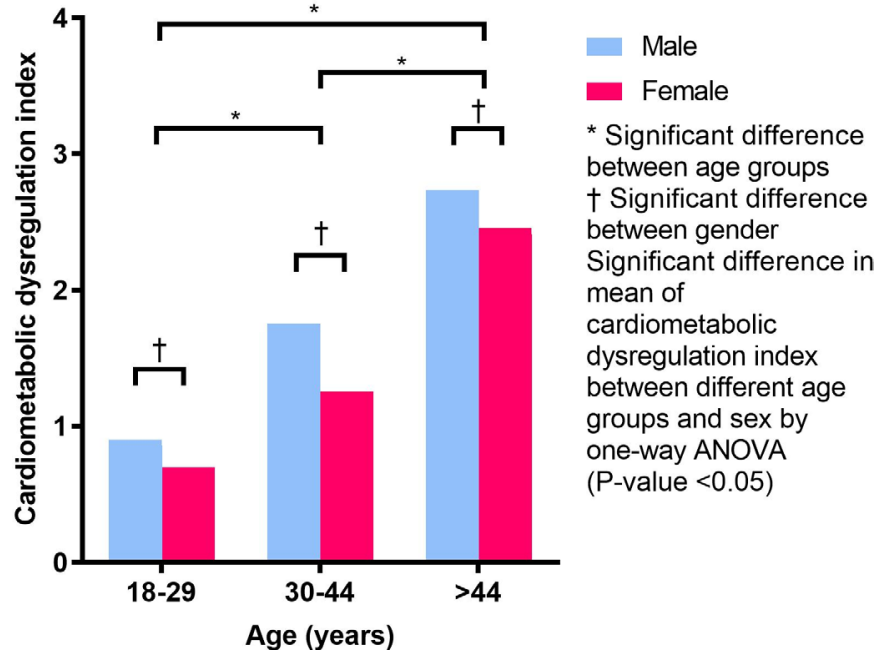

Figure 1 Cardiometabolic dysregulation index by gender and age groups. ANOVA, analysis of variance.

further longitudinal studies should be conducted to explore the causal relationship between sleep impairment, CMD, AL and adverse health outcomes using standardised questionnaires and polysomnography.

Our study also corroborates with the majority of previous studies that showed a positive relationship between smoking, CMD and AL. ${ }^{14}{ }^{31}$ Smoking is known to acutely impair glucose tolerance and insulin sensitivity, increase serum cholesterol and TG levels, and raise blood pressure, all of which have a direct effect on increasing $\mathrm{CMD}$ and $\mathrm{AL} .{ }^{55}$ Furthermore, consistent with previous studies, ${ }^{12} 133133$ 56-58 we confirmed that physical inactivity was associated with higher CMD. It has been observed that high level of PA was associated with low dysregulation of cardiovascular, metabolic and lipid axes and tended to be linked with low oxidative stress, ${ }^{13}$ supporting the role of PA in the primary prevention of diabetes, cardiovascular diseases and some cancers. However, different measurements had been used to assess PA level in different studies, rendering it difficult for clinicians to advise patients on how much PA would be required to achieve the desired health benefits. Most studies focused on the effect of leisure PA on AL, ${ }^{57} 58$ while the study by Petrovic et $a l^{13}$ used a non-validated self-reporting scale from 1 to 10 to assess participants' PA level on sedentary behaviour, manual work and sport practices to differentiate high versus low PA, which was not applicable in clinical setting. In our study, we used the GPAQ with its standardised scoring algorithm to account for the overall effect of PA from work, travelling and leisure activities on CMD, thereby improving the generalisability of our results to the working population. Meanwhile, our findings did not confirm an association between CMDI and alcohol consumption. When we categorised the participants' consumption pattern as harmful, non-harmful or none using the standardised AUDIT, we observed that non-harmful alcohol consumption was insignificantly associated with lower CMDI compared with nondrinkers, in line with the findings from Gallo et al and $\mathrm{Hu}$ et al studies. ${ }^{17} 18$ On the other hand, we failed to demonstrate an association between CMDI and vegetable and fruit intake as shown by Shi et $a l^{31}{ }^{31}$ suggesting the role of genetic and cultural factors underpinning the relationship between CMD and diet.

Table 3 Effect of lifestyle factors on cardiometabolic dysregulation by negative binomial regression

\begin{tabular}{|c|c|c|c|}
\hline Risk factors & IRR & $95 \% \mathrm{Cl}$ & $P$ value \\
\hline \multicolumn{4}{|l|}{ Smoking status (vs never smoked) } \\
\hline Former chronic smoker & 0.92 & 0.80 to 1.07 & 0.299 \\
\hline \multicolumn{4}{|l|}{ Drinking status (vs non-drinker) } \\
\hline Low-risk drinking (AUDIT score <8) & 0.94 & 0.86 to 1.04 & 0.225 \\
\hline Insufficient physical activity (vs sufficient physical activity) & 1.12 & 1.03 to 1.22 & $0.007^{*}$ \\
\hline \multicolumn{4}{|l|}{ Fruit/vegetable consumption per day (vs <3 servings) } \\
\hline $3-4$ servings & 0.97 & 0.87 to 1.07 & 0.523 \\
\hline$\geq 5$ servings & 1.03 & 0.88 to 1.21 & 0.706 \\
\hline Poor or fair sleep quality (vs good sleep quality) & 0.96 & 0.88 to 1.05 & 0.356 \\
\hline$\geq 9$ hours & 1.08 & 0.88 to 1.32 & 0.482 \\
\hline
\end{tabular}

IRR $>1$ indicates higher cardiometabolic dysregulation and vice versa.

Factors inside brackets are reference level.

*Significant difference at $\mathrm{p}<0.05$ by negative binomial regression adjusted by gender, age, marital status, educational level, household income, working status, physical comorbidities, mental comorbidities and regular health check.

AUDIT, AlcoholUse Disorders Identification Test; IRR, incidence rate ratio. 
The main strength of this study is that it is the first to explore CMD, an integral and important component of $\mathrm{AL}$, and its association with various HRBs in a large representative healthy adult population from a wide age range between 18 and 64 years, thereby providing good generalisability of results. Comprehensive data were collected on a wide range of socioeconomic and health behaviours, which allowed for adjustment of confounders. We must point out the limitation of the results from a cross-sectional study that does not allow the conclusion on causal relationship or examination on the association between the CMDI and the development of clinically important health outcomes such as diabetes, cardiovascular events and mortality. To examine CMDI as a predisease health index, we only excluded participants with self-reported established hypertension, diabetes, hyperlipidaemia and conditions which would directly affect the values of the biomarkers. Participants whose biomarkers reached the respective disease ranges in this cross-sectional assessment but were unaware of their condition might have been included in the study, because it would be inappropriate to confirm the diagnosis of hypertension, diabetes or hyperlipidaemia based on a single reading. Self-reported socioeconomic information and health behaviours may be susceptible to recall or social desirability bias. The ethnicity of the participants was not reported, although $97.5 \%$ of the participants reported being born in China. Lastly, measures of inflammatory and immunological dysregulation were not available from the data set; only CMD but not AL could be measured using the available biomarkers in this study. To further improve the predictive power of this clinically accessible CMDI, further study should consider adding measures of inflammatory and/or immunological activity on the currently proposed CMDI operationalisation and clinimetric criteria for identifying stressors and psychological distress and evaluating AL. ${ }^{59}$

\section{CONCLUSION}

In the Hong Kong healthy adult population, sleeping $<6$ hours, smoking, physical inactivity and higher number of HRBs were significantly associated with higher CMDI and potentially AL. Interventions targeting these modifiable HRBs should be widely advocated to prevent adverse health outcomes. Future longitudinal studies should be conducted to explore the temporal and causal relationship between HRBs, CMD, AL and adverse health outcomes, and the effectiveness of health behavioural interventions in reducing $\mathrm{CMD}$ and $\mathrm{AL}$ to prevent morbidities and mortalities.

Acknowledgements The authors wish to acknowledge the Department of Health (DH) of the Government of the Hong Kong Special Administrative Region for approval to perform this study and for provision of data.

Contributors EYTY and CLKL conceived and designed the study. EYTY, EHMT, CKHW, BMYC and CLKL were responsible for obtaining the data from the Department of Health and for data management. EYTY and CHNY conducted literature review. EYFW led the statistical analysis. EYFW and EHMT performed the statistical analysis. EYTY, EYFW, EHMT, CKHW, BMYC and CLKL contributed to the interpretation of the results. EYTY, CHNY and EHMT drafted the manuscript. EYFW, BMYC and CLKL provided critical revisions to the final draft. All authors approved the final manuscript for submission.

Funding The authors have not declared a specific grant for this research from any funding agency in the public, commercial or not-for-profit sectors.

Competing interests None declared.

Patient consent for publication Not required.

Ethics approval Ethics approval for this study was waived by the Institutional Review Board of the University of Hong Kong/Hospital Authority Hong Kong West Cluster on 30 July 2018 because the study only involved secondary analysis of anonymous data.

Provenance and peer review Not commissioned; externally peer reviewed.

Data availability statement All data relevant to the study are included in the article or uploaded as supplemental information.

Supplemental material This content has been supplied by the author(s). It has not been vetted by BMJ Publishing Group Limited (BMJ) and may not have been peer-reviewed. Any opinions or recommendations discussed are solely those of the author(s) and are not endorsed by BMJ. BMJ disclaims all liability and responsibility arising from any reliance placed on the content. Where the content includes any translated material, BMJ does not warrant the accuracy and reliability of the translations (including but not limited to local regulations, clinical guidelines, terminology, drug names and drug dosages), and is not responsible for any error and/or omissions arising from translation and adaptation or otherwise.

Open access This is an open access article distributed in accordance with the Creative Commons Attribution Non Commercial (CC BY-NC 4.0) license, which permits others to distribute, remix, adapt, build upon this work non-commercially, and license their derivative works on different terms, provided the original work is properly cited, appropriate credit is given, any changes made indicated, and the use is non-commercial. See: http://creativecommons.org/licenses/by-nc/4.0/.

\section{ORCID iDs}

Esther Yee Tak Yu http://orcid.org/0000-0001-7472-7083

Eric Yuk Fai Wan http://orcid.org/0000-0002-6275-1147

Carlos King Ho Wong http://orcid.org/0000-0002-6895-6071

\section{REFERENCES}

1 Johnson SC, Cavallaro FL, Leon DA. A systematic review of allostatic load in relation to socioeconomic position: poor fidelity and major inconsistencies in biomarkers employed. Soc Sci Med 2017;192:66-73.

2 Mauss D, Li J, Schmidt B, et al. Measuring allostatic load in the workforce: a systematic review. Ind Health 2015;53:5-20.

3 McEwen BS, Gianaros PJ. Central role of the brain in stress and adaptation: links to socioeconomic status, health, and disease. Ann N Y Acad Sci 2010;1186:190-222.

4 Beckie TM. A systematic review of allostatic load, health, and health disparities. Biol Res Nurs 2012;14:311-46.

5 Seeman TE, McEwen BS, Rowe JW, et al. Allostatic load as a marker of cumulative biological risk: MacArthur studies of successful aging. Proc Natl Acad Sci U S A 2001;98:4770-5.

6 Karlamangla AS, Singer BH, McEwen BS, et al. Allostatic load as a predictor of functional decline. MacArthur studies of successful aging. J Clin Epidemiol 2002;55:696-710.

7 Seeman TE, Singer BH, Rowe JW, et al. Price of adaptation-allostatic load and its health consequences. MacArthur studies of successful aging. Arch Intern Med 1997;157:2259-68.

8 McEwen BS. Mood disorders and allostatic load. Biol Psychiatry 2003;54:200-7.

9 Hwang A-C, Peng L-N, Wen Y-W, et al. Predicting all-cause and cause-specific mortality by static and dynamic measurements of allostatic load: a 10-year population-based cohort study in Taiwan. $J$ Am Med Dir Assoc 2014;15:490-6.

10 D'Alessio L, Korman GP, Sarudiansky M, et al. Reducing allostatic load in depression and anxiety disorders: physical activity and yoga practice as add-on therapies. Front Psychiatry 2020;11:501.

11 Rosemberg M-AS, Granner J, Li Y, et al. A scoping review of interventions targeting allostatic load. Stress 2020;23:519-28.

12 Suvarna B, Suvarna A, Phillips R, et al. Health risk behaviours and allostatic load: a systematic review. Neurosci Biobehav Rev 2020;108:694-711. 
13 Petrovic D, Pivin E, Ponte B, et al. Sociodemographic, behavioral and genetic determinants of allostatic load in a Swiss population-based study. Psychoneuroendocrinology 2016;67:76-85.

14 Crimmins EM, Kim JK, Seeman TE. Poverty and biological risk: the earlier "Aging" of the poor. J Gerontol A Biol Sci Med Sci 2009;64A:286-92.

15 Doan SN, Dich N, Evans GW. Childhood cumulative risk and later allostatic load: mediating role of substance use. Health Psychol 2014;33:1402-9.

16 Robertson T, Benzeval M, Whitley E, et al. The role of material, psychosocial and behavioral factors in mediating the association between socioeconomic position and allostatic load (measured by cardiovascular, metabolic and inflammatory markers). Brain Behav Immun 2015;45:41-9.

17 Gallo LC, Jiménez JA, Shivpuri S, et al. Domains of chronic stress, lifestyle factors, and allostatic load in middle-aged MexicanAmerican women. Ann Behav Med 2011:41:21-31.

18 Hu P, Wagle N, Goldman N, et al. The associations between socioeconomic status, allostatic load and measures of health in older Taiwanese persons: Taiwan social environment and biomarkers of aging study. J Biosoc Sci 2007;39:545-56.

19 Kusano $Y$, Crews DE, Iwamoto A, et al. Allostatic load differs by sex and diet, but not age in older Japanese from the GOTO islands. Ann Hum Biol 2016;43:34-41.

20 van Draanen J, Prelip M, Upchurch DM. Consumption of fast food, sugar-sweetened beverages, artificially-sweetened beverages and allostatic load among young adults. Prev Med Rep 2018:10:212-7.

21 Mattei J, Noel SE, Tucker KL. A meat, processed meat, and French fries dietary pattern is associated with high allostatic load in Puerto Rican older adults. J Am Diet Assoc 2011;111:1498-506.

22 Bei B, Seeman TE, Carroll JE, et al. Sleep and physiological dysregulation: a closer look at sleep Intraindividual variability. Sleep 2017;40.

23 Carroll JE, Irwin MR, Stein Merkin S, et al. Sleep and multisystem biological risk: a population-based study. PLoS One 2015;10:e0118467.

24 Chen X, Redline S, Shields AE, et al. Associations of allostatic load with sleep apnea, insomnia, short sleep duration, and other sleep disturbances: findings from the National health and nutrition examination survey 2005 to 2008. Ann Epidemiol 2014;24:612-9.

25 Clark AJ, Dich N, Lange T, et al. Impaired sleep and allostatic load: cross-sectional results from the Danish Copenhagen aging and midlife Biobank. Sleep Med 2014;15:1571-8.

26 Seeman T, Epel E, Gruenewald T, et al. Socio-Economic differentials in peripheral biology: cumulative allostatic load. Ann N Y Acad Sci 2010;1186:223-39.

27 Gersten O. Neuroendocrine biomarkers, social relations, and the cumulative costs of stress in Taiwan. Soc Sci Med 2008;66:507-19.

28 Seeman M, Stein Merkin S, Karlamangla A, et al. Social status and biological dysregulation: the "status syndrome" and allostatic load. Soc Sci Med 2014;118:143-51.

29 Gruenewald TL, Karlamangla AS, Hu P, et al. History of socioeconomic disadvantage and allostatic load in later life. Soc Sci Med 2012;74:75-83.

30 Gustafsson PE, Janlert U, Theorell T, et al. Socioeconomic status over the life course and allostatic load in adulthood: results from the Northern Swedish cohort. J Epidemiol Community Health 2011;65:986-92.

31 Shi L, Morrison JA, Wiecha J, et al. Healthy lifestyle factors associated with reduced cardiometabolic risk. Br J Nutr 2011;105:747-54.

32 Schmitz N, Deschênes SS, Burns RJ, et al. Cardiometabolic dysregulation and cognitive decline: potential role of depressive symptoms. Br J Psychiatry 2018;212:96-102.

33 Dipietro L, Zhang Y, Mavredes M, et al. Physical activity and cardiometabolic risk factor clustering in young adults with obesity. Med Sci Sports Exerc 2020;52:1050-6.

34 Lewis ME, Volpert-Esmond HI, Deen JF, et al. Stress and cardiometabolic disease risk for Indigenous populations throughout the lifespan. Int J Environ Res Public Health 2021;18. doi:10.3390/ ijerph18041821. [Epub ahead of print: 1302 2021].
35 Department of Health HKSAR Government. Report of population health survey 2014/2015. Hong Kong; 2017. https://www.chp.gov.hk/ files/pdf/dh_phs_2014_15_full_report_eng.pdf

36 Babor TF, Higgins-Biddle JC, Saunders JB. The alcohol use disorders identification test: guidelines for use in primary care. contract No.: WHO/MSD/MSB/01.6a. Geneva: WHO, 2001.

37 Department of Health HKSAR Government. Alcohol screening and brief intervention: a guide for use in primary care. Hong Kong, 2017.

38 World Health Organization. Global physical activity questionnaire (GPAQ). Geneva: World Health Organization, 2002.

39 World Health Organization. Global recommendations on physical activity for health. Geneva: World Health Organization, 2010.

40 World Health Organization. Diet, nutrition and the prevention of chronic diseases. Geneva: World Health Organization, 2003.

41 Itani O, Jike M, Watanabe N, et al. Short sleep duration and health outcomes: a systematic review, meta-analysis, and meta-regression. Sleep Med 2017;32:246-56.

42 Tan X, Chapman CD, Cedernaes J, et al. Association between long sleep duration and increased risk of obesity and type 2 diabetes: a review of possible mechanisms. Sleep Med Rev 2018;40:127-34.

43 Van Buuren S, Brand JPL, Groothuis-Oudshoorn CGM, et al. Fully conditional specification in multivariate imputation. J Stat Comput Simul 2006;76:1049-64.

44 Rubin DB. Multiple imputation for nonresponse in surveys. United States of America: John Wiley \& Sons, Inc, 1987

45 Feng L, Nian S, Tong Z, et al. Age-Related trends in lipid levels: a large-scale cross-sectional study of the general Chinese population. BMJ Open 2020;10:e034226.

46 Franklin SS, Gustin W, Wong ND, et al. Hemodynamic patterns of age-related changes in blood pressure. The Framingham heart study. Circulation 1997:96:308-15.

47 Joyner MJ, Wallin BG, Charkoudian N. Sex differences and blood pressure regulation in humans. Exp Physiol 2016;101:349-55.

$48 \mathrm{Ma} \mathrm{Q}$, Liu H, Xiang G, et al. Association between glycated hemoglobin $\mathrm{A} 1 \mathrm{c}$ levels with age and gender in Chinese adults with no prior diagnosis of diabetes mellitus. Biomed Rep 2016;4:737-40.

49 Lemieux I, Lamarche B, Couillard C, et al. Total cholesterol/HDL cholesterol ratio vs LDL cholesterol/HDL cholesterol ratio as indices of ischemic heart disease risk in men: the Quebec cardiovascular study. Arch Intern Med 2001;161:2685-92.

50 Berglund L, Brunzell JD, Goldberg AC, et al. Evaluation and treatment of hypertriglyceridemia: an endocrine Society clinical practice guideline. J Clin Endocrinol Metab 2012;97:2969-89.

51 McEwen BS, Karatsoreos IN. Sleep deprivation and circadian disruption: stress, allostasis, and allostatic load. Sleep Med Clin 2015;10:1-10.

52 Chaput J-P, McNeil J, Després J-P, et al. Seven to eight hours of sleep a night is associated with a lower prevalence of the metabolic syndrome and reduced overall cardiometabolic risk in adults. PLOS One 2013;8:e72832.

53 Prather AA, Leung CW. Association of insufficient sleep with respiratory infection among adults in the United States. JAMA Intern Med 2016;176:850-2.

54 Kalmbach DA, Arnedt JT, Song PX, et al. Sleep disturbance and short sleep as risk factors for depression and perceived medical errors in first-year residents. Sleep 2017;40. doi:10.1093/sleep/ zsw073. [Epub ahead of print: 0103 2017].

55 Frati AC, Iniestra F, Ariza CR. Acute effect of cigarette smoking on glucose tolerance and other cardiovascular risk factors. Diabetes Care 1996;19:112-8

56 Gay JL, Salinas JJ, Buchner DM, et al. Meeting physical activity guidelines is associated with lower allostatic load and inflammation in Mexican Americans. J Immigr Minor Health 2015;17:574-81.

57 Hampson SE, Goldberg LR, Vogt TM, et al. Using physiological dysregulation to assess global health status: associations with selfrated health and health behaviors. J Health Psychol 2009;14:232-41.

58 Upchurch DM, Rainisch BW, Chyu L. Greater leisure time physical activity is associated with lower allostatic load in white, black, and Mexican American midlife women: findings from the National health and nutrition examination survey, 1999 through 2004. Womens Health Issues 2015;25:680-7.

59 Guidi J, Lucente M, Sonino N, et al. Allostatic load and its impact on health: a systematic review. Psychother Psychosom 2021;90:11-27. 